lymph may be seen in the fascial sheaths. At first the surface is hard to the touch, but as the infection spreads, softening occurs and crepitation may be determined. Histologically the superficial necrotic muscle is composed of swollen fragmented fibers with separated perifibrillar sheaths embedded in a fibrous reticulum, in whose meshes are a large number of polymorphonuclear leukocytes, bacteria, red blood cells and débris. Some small blood clots lie in this area and examination showed them to be crowded with Bacillus aërogenes capsulatus, so that it is important to remove these so far as possible. Thrombosis of the vessels quickly occurs and their musculature undergoes lytic changes. In the area of dead muscle the color runs through reds to grays and greens and black. The tissue is no longer contractile. Gas forms late and pushes along the planes of fascia and pressure from this, in the case of such muscle as the tibialis posticus, with rigid fascial compartment, may be sufficient to partially occlude the main vessels. The gas may later burst into the muscle and find its way into the subcutaneous tissue. The authors found that massive thrombosis, apart from that resulting from trauma, is not concomitant of the process of gas gangrene. Histologically, in this area, myolysis was very general and the authors believe that this is due to the toxin of the organism. A fibrinous reticulum with its enmeshed leukocytes is less noticeable in the direction of spreading edge, its place being taken by fluid. The line of demarcation between living and dead muscle fibers is not sharp. Contractility of muscles returns in this area. In the area in which the muscle is contractile some of it may contain extensive blood extravasations. As regards the mode of spread of gangrene it occurs within the fascial sheath; thus the infection is confined to the affected muscle unless the main blood supply is interfered with or unless the exudate, tracking along the sheaths of arteries, nerves and tendons extends the process from one segment to another. In corroboration of this, authors record the case of a man whose penetrating shell wound of the thigh was opened twelve hours after being hit, and who, on the next morning, had constitutional signs of infection and a tympanitic thigh, the wound having the typical odor. The infection was found limited to the sartorious alone. Resection of the whole of this muscle was followed by recovery. In the line of demarcation a white line of fibrin and leukocytes abuts against the contractile muscle. Sloughing sometimes occurs at this line. The authors agree with Emery that toxins produced by the growth of these anaërobes and not the gas are the chief factors in the production of gangrene.

\title{
A Method for Producing a Rapid Immunity to Pneumococci.-
} Much attention has been given to the development of an active immunity to the pneumococcus. Attempts have been made to develop large quantities of antibody in animals for use as diagnostic and therapeutic sera. Various methods that have been used were developed by the individual investigators according to their conception of the important factors entering into the question of stimulating immune substances by pneumococci. Alexander (Jour. Med. Res., 1918, xxxvii, 471) undertook the investigation on the assumption that the immunity in pneumonia was related not alone to the presence of the pneumococcus but also to a reaction of the leukocytes. Pneumococci and leukocytes 
were mixed together and incubated for a certain length of time. The mixtures were then used as antigens and injected into rabbits, whose sera were tested for the presence of protective substances. The pneumococcus type II was used in the experiments. The organism was sensitized with immune serum, agglutinated and mixed in definite numbers with leukocytes. After incubation of the mixture it was inoculated intravenously into rabbits. Three daily injections were made and the blood was then tested six to eight days after the last injection. The rabbit serum was tested for protective substances in the white mouse. In this way a potent antipneumococcus serum was rapidly obtained in rabbits. Although ordinarily the rabbit is very susceptible to pneumococcus infection it was found that the sensitized pneumococcus mixed with leukocytes permitted the employment of large doses. The author did not study the manner of effect of the leukocytes upon the pneumococci. He particularly draws attention to the speed with which the protective substances appear in the serum of the treated animal.

\section{HYGIENE AND PUBLIC HEALTH}

ONDER THE CHARGE OF

MILTON J. ROSENAU, M.D.,

PROFESSOR OF PREVENTIVE MEDICINE AND HYGIENB, HARVARD MEDICAL SCHOOL; BOSTON, MASSACHUSETTS,

AND

GEORGE W McCOY, M.D.,

DIRECTOR OF HYGIENIC LABORATORY, ONITED STATES PCBLIC HBALTH BERVICE, WASHINGTON, D. C.

A Study of the Physical Condition of One Thousand Delinquents Seen in Court.-Anderson and Leonard (Boston Med. and Surg. Jour., June 13, 1918), state that the original purpose of their study was to determine what part, if any, routine physical examination might play in the disposition of a delinquent's case in court, and later in the institution of reconstructive measures while on probation. For this purpose the records of the last 1000 cases were taken from the files and the last physical condition of each person noted; a'so the relationship, if any, which the physical condition bore to his economic efficiency. Six hundred sixty-eight individuals, or 66.8 per cent. of the cases, were in good or fair health; 342 individuals, or 34.2 per cent. -1 out of every 3 persons-were in poor or bad health, and in such physical condition as to warrant urgent medical treatment. About 626, or 62.6 per cent., were considered self-supporting, while 374 , or 37.4 per cent., were not self-supporting. Some correlation between these figures is evident. Thirty-five per cent. of those found to be in good or fair physical condition had been steadily employed, while only 2 per cent. of those found to be in poor or bad physical condition had been steadily employed. Eighty-five per cent. of those found to be in good or fair 